\title{
Preface to the special issue on PKI
}

Published online: 19 December 2003 - @ Springer-Verlag 2003

It has been 25 years since Kohnfelder ("Towards a practical public-key cryptosystem", B.S. Thesis, MIT, May 1978) originally highlighted the need of having an infrastructure to support the application of public key cryptography. Such an architecture is commonly called a public key infrastructure (PKI). Many different PKIs have been proposed, especially over the last 10 years with the widespread application of public key cryptography to ecommerce in areas such as digital signatures and key management. Many issues both technical and legal have been identified with the use of PKI. In this special issue, new proposals for certificate structures and management are described to overcome some of the problems that have been identified with previous PKI systems.

One of the major bottlenecks in PKI is the requirement to query large databases in order to identify certificates that may have been revoked. Such a list is usually called a certificate revocation list (CRL). A new infrastructure architecture is proposed by Lopez, Mana, Montenegro, and Ortega that is based on the electronic mail service. They present an alternative solution to the use of CRLs, called Cert'eM. They show how Cert'eM can eliminate many of the problems with certificate revocation, as well as simplify the validation of certificates. A certificate revocation system based on the Merkle Hash Tree is presented by Munoz, Forne, Es- parza, and Soriano. Simulation details of this new system are given demonstrating improvements over previous proposals.

Another problem inherent with many PKI architectures is the time required for path construction and validation. A new certificate structure is proposed by Russell, called a recursive structure. Russell claims that this new design alleviates many of the difficulties with current X.509 certificates. Comparisons with conventional certificate procedures are made. Benefits and drawbacks of the new structure are highlighted.

One of the major issues with current PKI certificates is that of being user friendly. A new concept called a visible public key system is proposed by Laih and Chen. This system offers the potential of a more user-friendly public key certificate in that it provides an extra way for users to identify visually the owner of a public key.

A significant legal problem with PKI applied to digital signatures is the conflict between the normal use of cryptographically generated digital signatures and the centuries old approach to written signatures. A method to bridge this gap using trusted hardware is proposed by Blakley and Blakley.

Ed Dawson October 2003 